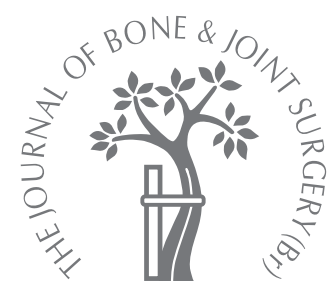

\title{
Correcting genu varum and genu valgum in children by guided growth
}

\author{
TEMPORARY HEMIEPIPHYSIODESIS USING TENSION BAND \\ PLATES
}

\author{
M. S. Ballal, \\ C. E. Bruce, \\ S. Nayagam \\ From The Royal \\ Liverpool Children's \\ NHS Trust, \\ Liverpool, England
}

\begin{abstract}
A total of 25 children (37 legs and 51 segments) with coronal plane deformities around the knee were treated with the extraperiosteal application of a flexible two-hole plate and screws. The mean age was $\mathbf{1 1 . 6}$ years (5.5 to 14.9$)$, the median angle of deformity treated was $8.3^{\circ}$ and mean time for correction was 16.1 months ( 7 to 37.3 ). There was a mean rate of correction of $0.7^{\circ}$ per month in the femur $\left(0.3^{\circ}\right.$ to $\left.1.5^{\circ}\right), 0.5^{\circ}$ per month in the tibia $\left(0.1^{\circ}\right.$ to $\left.0.9^{\circ}\right)$ and $1.2^{\circ}$ per month $\left(0.1^{\circ}\right.$ to $\left.2.2^{\circ}\right)$ if femur and tibia were treated concurrently. Correction was faster if the child was under 10 years of age $(p=0.05)$. The patients were reviewed between six and 32 months after plate removal. One child had a rebound deformity but no permanent physeal tethers were encountered.

The guided growth technique, as performed using a flexible titanium plate, is simple and safe for treating periarticular deformities of the leg.
\end{abstract}

Genu varum and valgum are common childhood deformities. Most improve spontaneously to the normal adult femorotibial angle before the age of eight years. ${ }^{1}$ Occasionally, deformities extend beyond the physiological limit to produce symptoms. These may be idiopathic in origin, or related to growth plate or bone forming disorders. Pain and limitation of activity may occur.

These deformities may be corrected by osteotomy and internal fixation or by gradual correction through external fixation. Gradual correction by hemiepiphyseal arrest is also possible using techniques such as stapling, ${ }^{2}$ percutaneous drill hemiepiphysiodesis ${ }^{3}$ or transphyseal screws. ${ }^{4}$ However, the risk of creating a permanent growth arrest means that these techniques have to be timed to take account of the size of deformity and remaining growth available. As this requires the use of growth charts, some error is inherent in the process and consequently underor over-correction may occur.

This prospective observational study describes the results of using a flexible titanium plate which corrects angular deformity by acting as a tension band on one side of the growth plate and offers the advantage of reversible hemiepiphyseal growth retardation. ${ }^{5}$

\section{Patients and Methods}

We treated 25 consecutive children (51 physes, 12 bilateral procedures) with symptomatic varum or genu valgum by guided growth using a flexible two-hole titanium plate (8-plate; Orthofix SRL, Verona, Italy). All the children were entered into a database and reviewed as outpatients at four-monthly intervals until correction was complete (Fig. 1 ).

A decision to offer surgical correction was based on symptoms and absence of spontaneous improvement after observation for at least 12 months. The deformity was assessed using a standing scanogram which enabled femorotibial angles and the position of the mechanical axis to be measured. We considered the mechanical axis of the limb to be abnormal if it crossed the knee joint outside the inner two quadrants of a six quadrant zone (Fig. 2). ${ }^{6}$ In order to determine if there was sufficient growth remaining for correction by guided growth, a bone age was obtained in all patients. ${ }^{7}$ Those who were within six months of skeletal maturity (14 years of bone age for females and 16 years for males), were considered unsuitable for this technique. ${ }^{8}$

Operative technique. The level of the physis on the relevant side and segment (distal femur or proximal tibia) was identified using fluoroscopy. The centre of the physis was estimated by palpating the anterior and posterior margins of the femur or tibia and placing a $2 \mathrm{~cm}$ skin incision over this position. The fascia lata was divided longitudinally. The periosteal surface was exposed by blunt dissection, taking care not to injure this layer and the perichondrial ring. The plate was placed over the physis and 


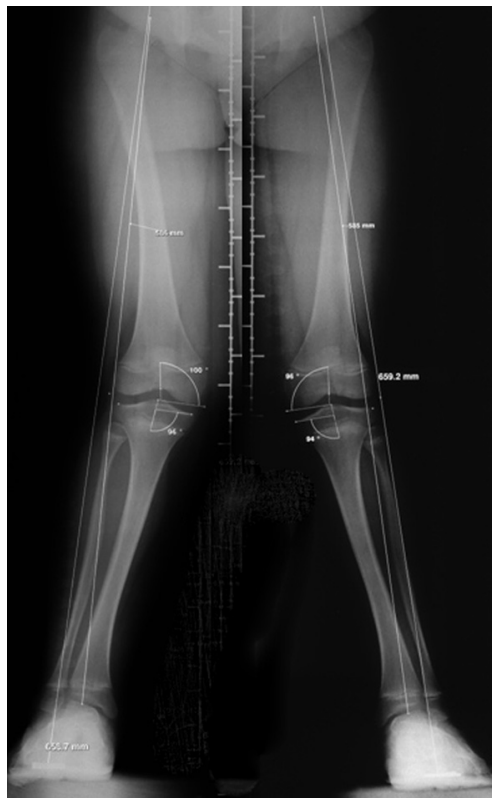

Fig. 1a

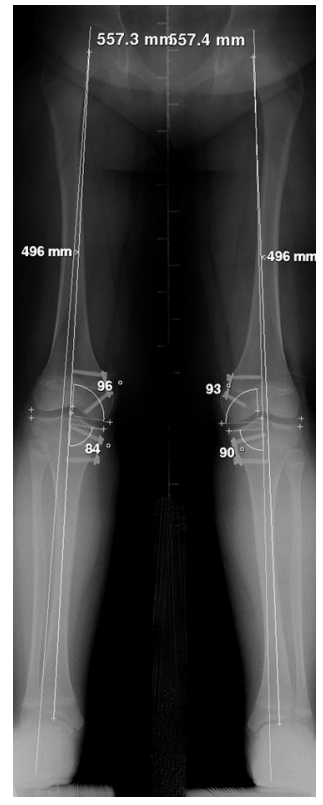

Fig. 1b
Scanogram showing a) bilateral genu-valgum and b) with correction 15 months after treatment with titanium plates and screws at the distal femur and at the proximal tibia. The length measurements indicate total length of the limb (from the roof of the acetabulum to a heel marker) and the length of the mechanical axis. The angles are the medial distal femoral and medial proximal tibial angles.

provisionally secured with a hypodermic needle through a small central hole in the plate. Satisfactory positioning was confirmed by fluoroscopy. Threaded guide wires were then driven through the centres of the two main holes of the plate, aiming to keep the direction of these wires parallel to the physis. The cortex was broached using a cannulated drill and a self-tapping $4.5 \mathrm{~mm}$ titanium screw was passed over the guide wire. These wires were extracted before each screw was finally secured onto the plate and its position checked. A compression bandage was applied after wound closure.

Patients were discharged on the following day having safely mobilised partially weight-bearing on crutches. The compression bandage was reduced after three or four days and knee motion encouraged. Full weight-bearing was usually achieved in the second week.

Patients were monitored clinically and radiologically at four-monthly intervals. When clinical correction of the deformity was deemed satisfactory, a standing scanogram was obtained to confirm the clinical impression. The desired correction was restoration of the mechanical axis to within the inner two zones of a six-zone division of an anteroposterior radiograph of the knee; when this occurs, there is improvement ${ }^{9}$ in the position of the ground reaction forces (Fig. 2). Plate removal was undertaken as day surgery and the patients followed up until skeletal maturity to check for rebound deformity, limb-length discrepancy or premature physeal closure.

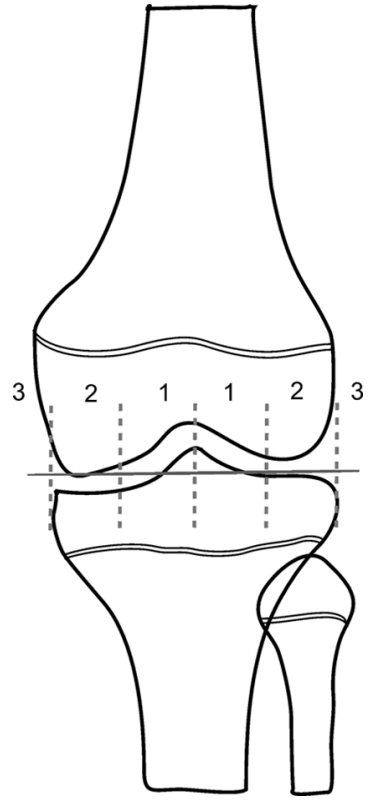

Fig. 2

Drawing showing the six zone division of the knee. The correction is deemed satisfactory if the mechanical axis of the limb shifts to within the inner two central zones of an anteroposterior radiograph of the knee. This improves the position of the ground reaction force through the knee. ${ }^{23}$

Statistical analysis. Data were summarised using means and ranges. A tibiofemoral angle of $6^{\circ}$ was used as the normal from which the size of deformity was measured. The patients were divided into two groups; those aged $<10$ years and those older. This was thought to represent a mean threshold between those pre-pubertal and those postpubertal. Further analysis was performed using linear regression methods to assess the relationship between age and rate of correction. Statistical significance was set at $5 \%$ (p-value of 0.05).

\section{Results}

The 25 children included in the study had a mean follow-up of 12.4 months (6 to 32) after plate removal. Those who had not reached skeletal maturity at the time of the study will be followed up until maturity. There were 10 females and 15 males. The mean age was 11.6 years (5.5 to 14.9).

There were 12 bilateral and 13 unilateral deformities. The pre-operative deformity ranged from $53^{\circ}$ of genu varum to $17^{\circ}$ of genu valgum. The mean deformity for genu varum was $28.8^{\circ}\left(11.1^{\circ}\right.$ to $\left.53.3^{\circ}\right)$ and $8.4^{\circ}\left(3^{\circ}\right.$ to $\left.25^{\circ}\right)$ for genu valgum. Owing to the large range in size and direction of deformity, there was a correspondingly large range in the time required for gradual correction; the mean was 


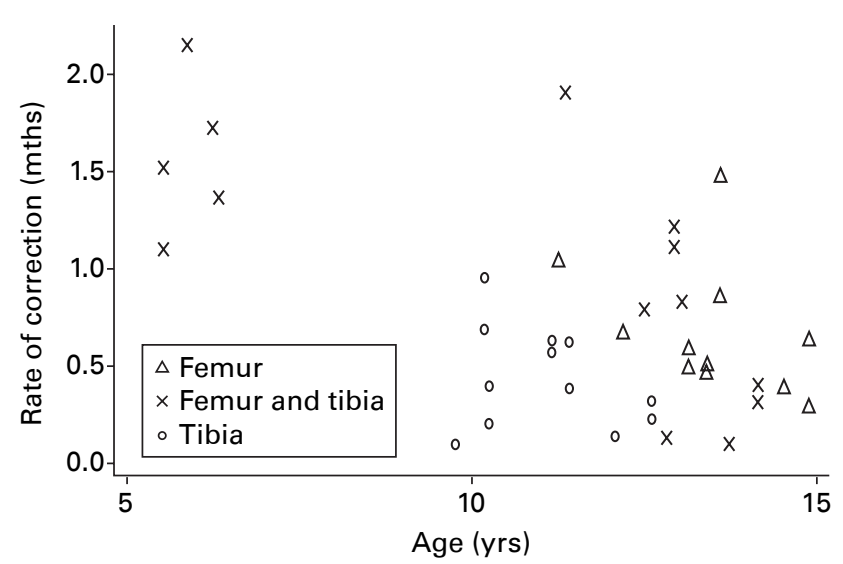

Fig. 3a

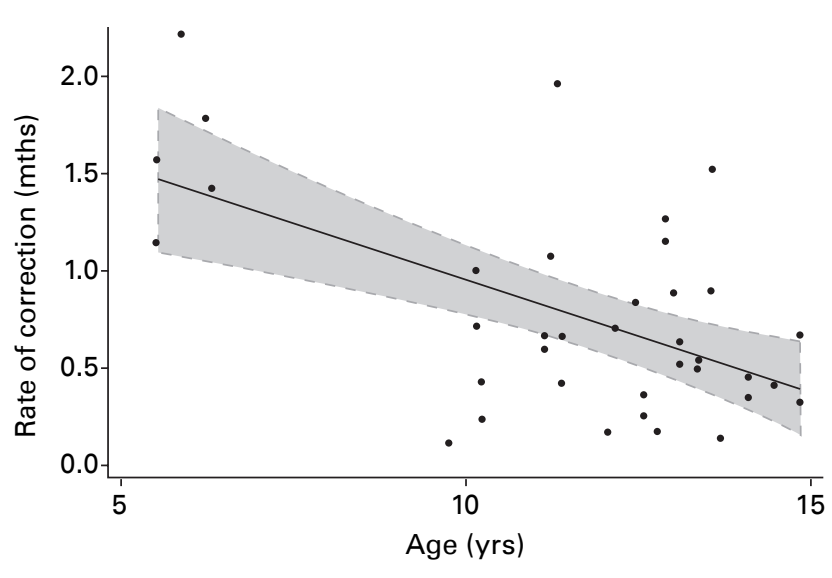

Fig. 3b

Graph showing a) rate of correction of deformity in relation to limb segment and age, and b) regression analysis of the overall speed of correction against the child's age. The estimated regression line is shown as the $95 \%$ confidence interval for the estimate (shaded area).

16.1 months (7 to 37.3 ). The rate of correction was influenced by the physis treated and the age of the child. There was a mean rate of correction of $0.7^{\circ}\left(0.3^{\circ}\right.$ to $\left.1.5^{\circ}\right)$ per month in the distal femur, of $0.5^{\circ}\left(0.1^{\circ}\right.$ to $\left.1.0^{\circ}\right)$ per month in the proximal tibia and of $1.2^{\circ}\left(0.1^{\circ}\right.$ to $\left.2.2^{\circ}\right)$ per month if femur and tibia were treated concurrently (Fig. 3a).

Regression analysis of the overall rate of correction against the child's age showed an expected decrease as the child got older (Fig. 3b). When the sample was divided into two groups to reflect an approximate age of onset of puberty, the overall rate of correction for children under the age of 10 years was $1.4^{\circ}$ per month, compared with $0.6^{\circ}$ per month for the older children $(\mathrm{p}=0.05)$.

There were complications in two patients, namely radiological evidence of plate and screw migration in the first and deep infection in the second. In the former the plate and screws were removed and replaced. In the latter, a twostage exchange of metalwork was carried out with an interval of six weeks of antibiotic therapy to treat the deep infection. The new plate was re-inserted and provided correction of deformity without further complications.

There was one rebound deformity in a five-year-old child who had a unilateral genu valgum deformity of $31^{\circ}$. Plates were inserted over the medial distal femoral and proximal tibial physes. Correction was accomplished in 11.5 months to a femorotibial angle of $5.3^{\circ}$ and the plate was removed. Six months later a rebound deformity to $9.7^{\circ}$ valgus was measured radiologically. At last follow-up, 20 months after plate removal, the deformity had increased and further surgical correction is being considered.

\section{Discussion}

Bony deformity may be treated by osteotomy, for which careful analysis of the deformity and detailed operative planning are essential. Analysis of the deformity has been formalised and clarified by the centre of rotation angula- tion method. ${ }^{10}$ However, in deformities around a joint, an open physis can make correction technically difficult. Furthermore, osteotomy and acute correction of deformities carry a risk of complications such as compartment syndrome and nonunion. ${ }^{11,12}$

Bony deformity may also be corrected by manipulating the growth behaviour of an open physis. ${ }^{12,13}$ In experimental and clinical work, Haas ${ }^{13,14}$ demonstrated the resilience of the physis following surgical instrumentation. He documented growth inhibition by placing a wire loop around a canine distal femoral physis and noted that growth resumed when the wire broke. Based on this work and others, ${ }^{14,15}$ procedures have been introduced to manipulate the growth from a physis. The technique of hemiepiphysiodesis is well established in children, in particular the use of staples and transphyseal screws. Both methods exert compression on the physis and, when placed eccentrically, can retard development on the side of application and thereby produce asymmetrical growth. ${ }^{2,4,15}$ Some concerns have been raised over the use of staples in younger children; the prolonged presence of rigid implants bracketing the physis has prompted worries over excessive compression leading to permanent physeal closure. ${ }^{16,17}$ Aykut et al ${ }^{18}$ demonstrated clinically and experimentally that transgression of the periosteum during staple insertion or removal poses a risk of producing a physeal bar. In contrast, a recent study demonstrated that percutaneous epiphysiodesis using transphyseal screws in the correction of lower limb deformities in younger children can be a reversible method. ${ }^{19}$

The flexible plate concept developed by Stevens ${ }^{5}$ differs from staple or screw compression across the physis. The placement of this non-rigid tension band device at the perimeter of the physis produces the effect of a focal hinge. While some compression is applied across the physis, it is not constant owing to the propensity of the screws to diverge as correction proceeds. Once the screws reach their 
maximum divergence, there is also the facility within the flexible plate to bend. Both of these features reduce the risk of creating a permanent physeal tether through excessive and prolonged compression across the physis. Also, only one plate per physis is needed to produce correction, whereas three staples per physis are usually needed for the same effect. This clinical study also confirms the reversible nature of growth inhibition produced by the device.

The speed of correction in hemiepiphysiodesis is determined by the nature of the growth modulation (staple, transphyseal screw or flexible plate), the age of the child and the physis treated. Burghardt et $\mathrm{al}^{20}$ described the rate of correction using the flexible plate method in terms of mechanical axis deviation. In this case, the rate of improvement in the mechanical axis derived from the tibial segment was slower than from the femoral segment. Conversely, we can report on rates of correction as derived from each physis in terms of angular improvement per month, but also describe the influence of age on the process. The measurement of the rates of correction per month allows the surgeon to estimate the overall treatment time and provide parents with relevant information. Visual appreciation of the effect of gradual correction usually occurs towards the end of the treatment period. Providing counselling about the estimated time needed will allay any parental anxiety over what may appear initially to be a lack of progress.

Implant migration was noted in one patient. and this has also been documented with the use of staples. ${ }^{16,21}$ This complication may have been due to a technical error in the primary procedure, as the distal screw was placed very close to the physis and would have been avoided through selection of a longer plate to bridge the growth plate. There were no instances of screw or implant back-out as can occur with staples. ${ }^{22,23}$

The average age of the children in this study was 11.6 years with a $25 \%$ to $75 \%$ interquartile range of 10 to 13 years. Therefore, the majority were adolescents. This is similar to the study by Stevens. ${ }^{5}$ However, no previous report has been able to estimate a rate of correction by physeal level or age using this treatment method. We found the fastest rates of correction were achieved when the distal femoral and proximal tibial physes were treated concurrently, or when the technique was used in children under the age of ten years. The ability to harness this technique for younger children, as in some of the cases in this study, is a major advantage and reduces the need for osteotomy for deformities around the knee joint. The only caveat is that the physis itself must be normal and respond to growth guidance through the flexible plate. Finally, the ability to perform the implantation and removal of the device as day surgery or with overnight stay is an added advantage over correction by osteotomy. Most children were walking confidently without aids by the second post-operative week.
This study has confirmed the reversible nature of growth inhibition on the physis produced by a bridging flexible titanium plate and has demonstrated its value in correcting deformities around the knee. The procedure is technically simple and has significant potential for treating genu varum and valgum for many children without the need for osteotomy.

The author or one or more of the authors have received or will receive benefits for personal or professional use from a commercial party related directly or indirectly to the subject of this article. In addition, benefits have been or will be directed to a research fund, foundation, educational institution, or other nonprofit organisation with which one or more of the authors are associated.

\section{References}

1. Salenius P, Vankka E. The development of the tibiofemoral angle in children. $J$ Bone Joint Surg [Am] 1975;57-A:259-61.

2. Blount W. A mature look at epiphyseal stapling. Clin Orthop 1971;77:158-63.

3. Bowen JR, Torres R, Forlin E. Partial epiphysiodesis to address genu varum or genu calgum. J Pediatr Orthop 1992;12:359-64.

4. Metaizeau J-P, Wong-Chung J, Bertrand H, Pasquier P. Percutaneous epiphysiodesis using transphyseal screws (PETS). J Pediatr Orthop 1998;18:363-9.

5. Stevens PM. Guided growth for angular correction: a preliminary series using a tension band plate. J Pediatr Orthop 2007;27:243-59.

6. Stevens PM, Pease F. Hemiepiphysiodesis for posttraumatic tibial valgus. J Pediatr Orthop 2006;26:385-92.

7. Blair V, Walker S, Sheridan J, Schoenecker PL. Epiphysiodesis: a problem of timing. J Pediatr Orthop 1982;2:281-4.

8. Anderson M, Green WT, Messner MB. Growth and predictions of growth in the lower extremities. J Bone Joint Surg [Am] 1963;45-A:1-14.

9. Stevens PM, MacWilliams B, Mohr RA. Gait analysis for genu valgum. J Pediatr Orthop 2004;24:70-4.

10. Paley D. Principles of deformity correction. Berlin: Springer, 2002

11. Mycoskie PJ. Complications of osteotomies about the knee in children. Orthopaedics 1981;4:1005-15.

12. Steel HH, Sandrow RE, Sullivan PD. Complications of tibial osteotomy in children for genu varum or valgum: evidence that neurological changes are due to ischemia. $J$ Bone Joint Surg [Am] 1971;53-A:1629-35.

13. Haas SL. Retardation of bone growth by a wire loop. J Bone Joint Surg 1945;27:2536 .

14. Haas SL. Mechanical retardation of bone growth. J Bone Joint Surg [Am] 1948;30A:506-12.

15. Blount WP, Clarke GR. Control of bone growth by epiphyseal stapling: a preliminary report. J Bone Joint Surg [Am] 1949;31-A:464-78.

16. Bylski-Austrow DI, Wall EJ, Rupert MP, Roy DR, Crawford AH. Growth plate forces in the adolescent human knee: a radiographic and mechanical study of epiphyseal staples. J Pediatr Orthop 2001;21:817-23.

17. Farnum CE, Nixon A, Lee A0, et al. Quantitative three-dimensional analysis of chondrocytic kinetic responses to short-term stapling of the rat proximal tibial growth plate. Cells Tissues Organs 2000;167:247-58.

18. Aykut US, Yazici M, Kandemir U, et al. The effect of temporary hemiepiphyseal stapling on the growth plate: a radiologic and immunohistochemical study in rabbits. J Pediatr Orthop 2005;25:336-41.

19. Khoury J, Tavares J, McConnell S, Zeiders G, Sanders J. Results of screw epiphysiodesis for the treatment of limb length discrepancy and angular deformity. $J$ Pediatr Orthop 2007;27:623-8.

20. Burghardt RDE, Herzenberg JE, Standard SC, Paley D. Temporary hemiepiphyseal arrest using a screw and plate device to treat knee and ankle deformities in children: a preliminary report. J Child Orthop 2008;2:187-97.

21. Zuege RC, Kempken TG, Blount WP. Epiphyseal stapling for angular deformity at the knee. J Bone Joint Surg [Am] 1979;61-A:320-9.

22. Brockway A, Craig WA, Cockreli BR Jr. End-result study of sixty-two stapling operations. J Bone Joint Surg [Am] 1954;36-A:1063-70.

23. Fraser RK, Dickens DR, Cole WG. Medial physeal stapling for primary and secondary genu valgum in late childhood and adolescence. J Bone Joint Surg [Br] 1995;77B:733-5. 